\title{
Drug-susceptibility testing in tuberculosis: methods and reliability of results
}

\author{
S.J. Kim
}

ABSTRACT: The demand for reliable drug-susceptibility testing (DST) increases with the expansion of antituberculosis drug-resistance surveillance, and with the need for an appropriate treatment of multidrug-resistant tuberculosis, whose incidence gradually increases in many parts of the world. However, the reliability of DST results obtained through widely used methods does not meet acceptable levels, except for DST to isoniazid and rifampicin.

In general, susceptibility results are highly predictable, while resistance results show low predictive values when the resistance prevalence is $<10 \%$. Poor reliability stems from a weak correlation with clinical response and a low reproducibility due to the poor standardisation of the complex and fragile test procedures. Therefore, in vitro criteria of resistance for susceptibility testing should be carefully determined with representative clinical samples of Mycobacterium tuberculosis isolated from patients never treated with any antituberculosis drug, and from patients having failed treatment with a regimen containing the tested drug; DST should then be carefully standardised to obtain reproducible results.

The critical concentration of some drugs is close to the minimal inhibitory concentration for wild susceptible strains and, thus, drug-susceptibility testing is prone to yield poorly reproducible results. These issues call for physicians' attention when using the results from drug-susceptibility testing for case management.

\section{KEYWORDS: Drug resistance, drug-susceptibility testing, tuberculosis}

$\mathrm{n}$ many countries, the wide use of the standard short-course regimen has led to an increasing incidence of multidrug-resistant (MDR) tuberculosis (TB), defined as resistance to at least isoniazid (INH) and rifampicin (RFP) [13]. Significant high rates of MDR-TB were observed in some parts of the world, not only among previously treated TB patients, due to poor case management, but also among new cases due to transmission in the community. The situation has turned into a pressing demand for drug-susceptibility testing (DST) in order to accomplish drug-resistance surveillance (DRS), and also to develop efficient regimens for appropriate treatment of individual cases.

As a result of inappropriate and/or inadequate treatment, drug resistance emerges by selective multiplication of resistant mutants within the lesions, in spite of the presence of growthinhibitory concentrations of a drug. The frequency of drug-resistant mutants and their resistance levels vary depending on the drug and the mutated genes and sites, whose phenotypic expressions include the following: alterations of the binding site of drug-target molecules; loss of enzymes activating drug molecules; permeability changes to the drug, including efflux; and production of drug-inactivating enzymes, such as $\beta$ lactamase. There are a variety of methods to determine the susceptibility of Mycobacterium tuberculosis to antituberculosis drugs, but none of them is perfect, and their results do not satisfy clinicians for the effective treatment of TB patients.

Most of the currently used DST methods suffer from low predictability associated with clinical irrelevance of the results and from unacceptable

\section{AFFILIATIONS}

International Union Against

Tuberculosis and Lung Disease,

Paris, France.

CORRESPONDENCE

S.J. Kim

International Union Against

Tuberculosis and Lung Disease

101-703 Unjeongmaul

Guseongup

Yonginsi

Kyeonggido 449-560

South Korea

Fax: 82313044301

E-mail: SJKim@iuatld.org

Received:

September 252004

Accepted:

October 062004 
low reliability resulting from poor reproducibility. This review focuses on general features of DST methods concerning the clinical relevance and the reproducibility of the technique.

\section{DRUG-SUSCEPTIBILITY TESTING METHODS}

Drug susceptibility of $M$. tuberculosis can be determined either by observation of growth or metabolic inhibition in a medium containing antituberculosis drug, or by detection, at the molecular level, of mutations in the genes related to drug action. From a technical standpoint, drug susceptibility is determined on the basis of growth (or metabolic) inhibition induced by the drug by means of: 1) macroscopic observation of growth in drug-free and drug-containing media; 2) detection or measurement of the metabolic activity or products; 3 ) lysis with mycobacteriophage; and 4) detection of genetic mutations using molecular techniques.

Conventional culture methods using egg- or agar-based media are still the most utilised in many countries $[4,5]$. Although the long turnaround time of DST results displeases physicians for the purpose of case management, it is suitable for DRS. The standard methods using Löwenstein-Jensen medium include the proportion method, the absolute concentration method and the resistant ratio method, which are fairly well standardised with clinical samples, at least for the major antituberculosis drugs [4]. Among conventional methods, the proportion method is the most preferred choice, but the absolute concentration method is also commonly used on account of its technical simplicity for inoculum preparation and for reading the results.

In order to shorten the turnaround time and make it more convenient for case management, numerous new techniques have appeared, aiming to detect growth inhibition as early as possible. The most commonly used systems are detection of $\mathrm{CO}_{2}$ production, such as BACTEC 460 [6] or MB/Bact [7], and oxygen consumption, such as Mycobacteria Growth Indicator Tube [8]; there are others in developmental stage, such as oxidation-reduction indicators like resazurin or tetrazolium bromide [9, 10] and the phage-based techniques [11, 12]. Particle-counting immunoassay [13] can also curtail turnaround times by detecting a low-level multiplication of $M$. tuberculosis. Many of those new techniques are difficult to implement in the countries where they are needed the most, because of high costs, technical complexity and absence of appropriately trained human resources. In addition, they still need clinical evaluation to verify their claimed efficiency under various settings. Most of all, none of these techniques has been well calibrated with representative clinical samples of $M$. tuberculosis in order to determine the clinically relevant criteria of resistance (i.e. cut-off points).

There are numerous reports on molecular techniques to detect gene mutations related to resistance, including hybridisation [14-20] of amplified gene segments or other PCR-based methods. However, not all resistance-related genes for the different antituberculosis drugs and their sites of mutation have been found, except for rpoB gene mutations, which lead to RFP resistance. These molecular techniques normally require primary amplification, and, therefore, when they are used on a routine basis for long periods of time, they are not free from false results due to contaminating amplicons and/or chromosomal DNA.

\section{RELIABILITY OF DRUG-SUSCEPTIBILITY TEST RESULTS}

The World Health Organization and the International Union Against Tuberculosis and Lung Disease established the Supranational TB Reference Laboratory Network through which they intend to assure DST proficiency testing in countries implementing DRS [1-3]. Proficiency testing results from nine rounds, undertaken during 1994-2002, showed that average sensitivities to detect resistance to INH, RFP, streptomycin (SM) and ethambutol (EMB) were 98.7\%, 97.2\%, $90.8 \%$ and $89.3 \%$, respectively [3]. The corresponding sensitivities to detect susceptibility to INH, RFP, SM and EMB were $98.5 \%, 96.8 \%, 93.9 \%$ and $94.0 \%$, respectively [3]. The predictive values of the susceptibility DST results, which have been calculated on the basis of the aforementioned figures, were high for all four drugs (>93\%), even at $40 \%$ prevalence, while predictive values of the resistance DST results varied greatly between the four major drugs (figs 1 and 2). Below $10 \%$ prevalence of resistance, the predictive values for EMB and SM were significantly lower than those for INH and RFP. The predictive value of resistance, at the median prevalences of resistance to specific drugs among the pulmonary $\mathrm{TB}$ patients, as has been previously published [3], was $80.6 \%$ for INH at a $5.9 \%$ median resistance prevalence, $30.4 \%$ for RFP at $1.4 \%, 50.0 \%$ for SM at $6.3 \%$, and $10.4 \%$ for EMB at $0.8 \%$. Among previously treated cases, predictive values were, of course, higher due to higher median prevalence of resistance, as follows: $91.6 \%$ for $\mathrm{INH}$ at a $14.4 \%$ median prevalence; $74.6 \%$ for RFP at $8.4 \%$; $65.8 \%$ for SM at $11.4 \%$; and $34.8 \%$ for $\mathrm{EMB}$ at $3.5 \%$. It is clear that resistance results for EMB and SM are less reliable than those for INH and RFP. Similar findings were obtained through rapid growthdetection systems [8].

\section{FACTORS INFLUENCING DRUG-SUSCEPTIBILITY TEST RESULTS}

DST methods and the interpretation of their results vary greatly; however, it is noteworthy that, in order to obtain

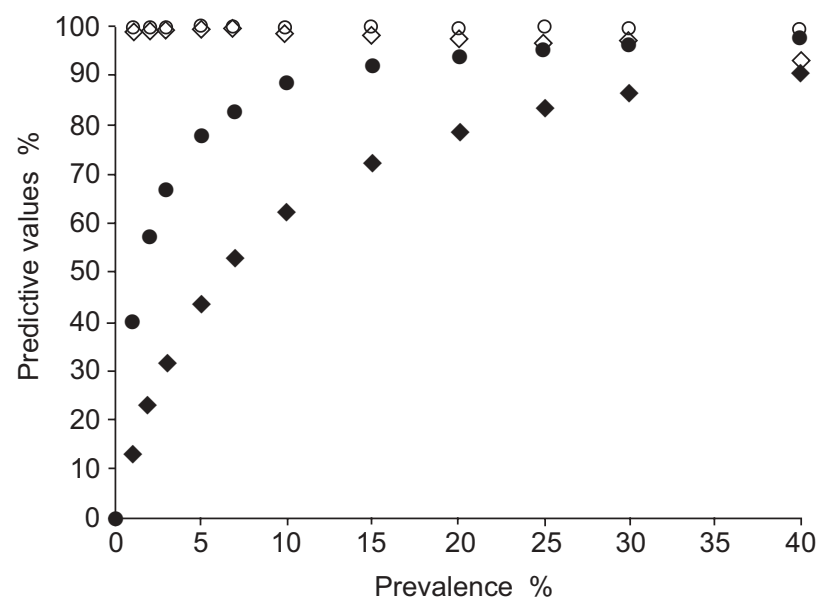

FIGURE 1. Predictive values of isoniazid (INH) and streptomycin (SM) susceptibility testing results. $\bigcirc$ : INH susceptibility; - : INH resistance; $\diamond:$ SM susceptibility; : SM resistance. Data taken from [3]. 


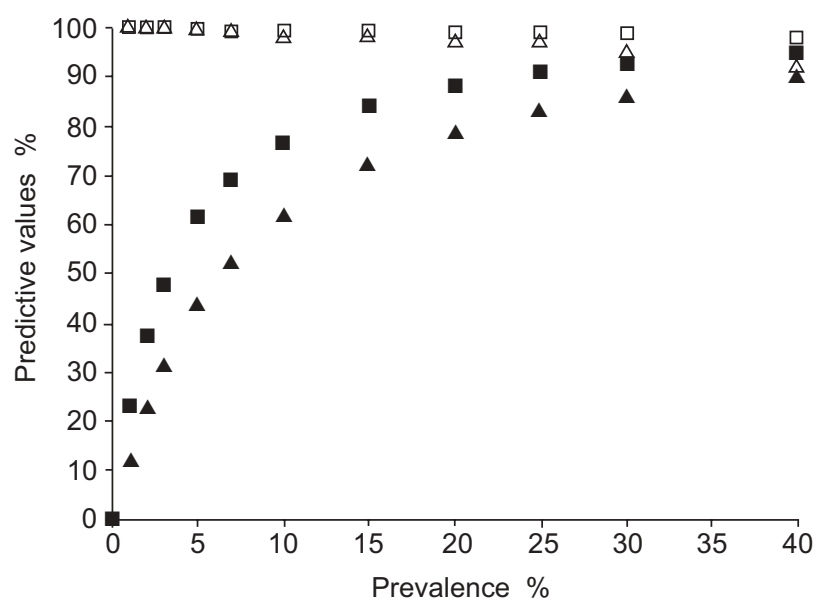

FIGURE 2. Predictive values of rifampicin (RFP) and ethambutol (EMB) susceptibility testing results. $\square$ : RFP susceptibility; $\mathbf{\text { a }}$ RFP resistance; $\triangle$ : EMB susceptibility; $\mathbf{\Lambda}$ : EMB resistance. Data taken from [3]

clinically relevant and dependable results for case management, the probability of misclassification of clinically susceptible or clinically resistant strains must be minimal or absent. Aiming to meet this requirement, it has been suggested to fix the criteria at the minimum inhibitory concentration (MIC) and $1 \%$ of the critical resistant proportion of $M$. tuberculosis strains isolated from never-treated patients [4, 21]. However, primary drug resistances, including natural resistance, would interfere with this approach. Another method would be the comparison of clinical responses of strains bearing all the possible levels of resistance, in order to find the clinically relevant critical level of resistance. This approach may seem ideal, but it is not realistic. For this reason, it is recommended to determine in vitro criteria, which could be used to predict clinical resistance and susceptibility with acceptable accuracy, by testing well-defined and representative clinical strains of $M$. tuberculosis [4, 22] isolated from patients treated under well-controlled and properly recorded clinical settings. The DST method under evaluation should be calibrated by comparing the MIC of

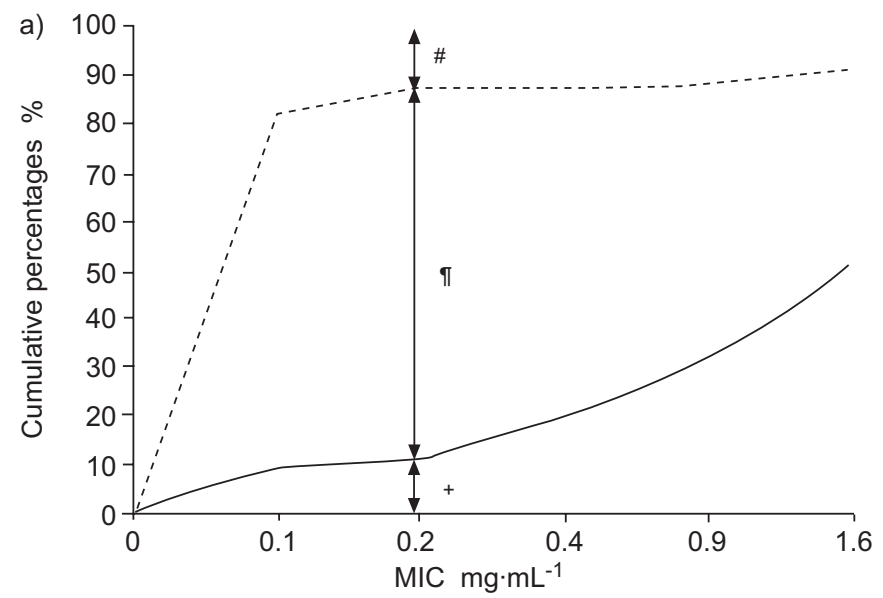

probably susceptible (PS) strains, derived from never-treated patients, with the MIC of probably resistant (PR) strains, derived from patients who apparently had treatment failure with regimens containing the corresponding drug. The difference in cumulative susceptible percentages between PS and PR clinical isolates (at various concentrations) was greater at $0.2 \mu \mathrm{g} \cdot \mathrm{mL}^{-1}$ with INH and at $40.0 \mu \mathrm{g} \cdot \mathrm{mL}^{-1}$ with RFP [23]; discrimination power values were $75.8 \%$ and $85.8 \%$, respectively (figs 3 and 4 ). At the same concentrations, the $1 \%$ critical resistant proportion could discriminate PS and PR most reasonably for both drugs, showing a discrimination power of $77.1 \%$ for INH and $85.6 \%$ for RFP [23]. MitCHISON [24] found that SM-resistant strains were discriminated from susceptible strains at $16 \mu \mathrm{g} \cdot \mathrm{mL}^{-1}$ with a difference of $52.4 \%$. LEFFORD and MITCHISON [22] reported that ethionamide (ETH) resistance was discriminated at $40.0 \mu \mathrm{g} \cdot \mathrm{mL}^{-1}$ with a $46.7 \%$ difference. It is also interesting to notice that most reasonable criteria of resistance could be different between laboratories, even when they apply the same method. EMB PR strains have been discriminated from PS strains at $2 \mu \mathrm{g} \cdot \mathrm{mL}^{-1}$ in the laboratory of the Korean Institute of Tuberculosis, Seoul, South Korea, while they were better distinguished at $4 \mu \mathrm{g} \cdot \mathrm{mL}^{-1}$ in the laboratory of the Royal Postgraduate Medical School, London, UK, using the same medium and method, and testing the same PR strains (personal communication, D.A. Mitchison, Medical Research Council Unit for Laboratory Studies of Tuberculosis, Royal Postgraduate Medical School, Hammersmith Hospital, London, UK; fig. 5). These data seem to indicate that differences in critical concentration might have resulted from different susceptibility levels of PS strains and disparities in the test environment. Resistance levels of PR strains vary greatly, except to RFP; therefore, it is not possible to determine a reasonable cut-off point without a careful calibration of susceptibility with PS strains. Those studies clearly show that the discrimination between PR and PS strains is more reliable for INH and RFP than for other drugs. It is also clear that the development of a DST method capable of distinguishing PR and PS strains at $100 \%$ is, in fact, not feasible because of the technical variations resulting from the physicochemically labile environment of the test.

b)

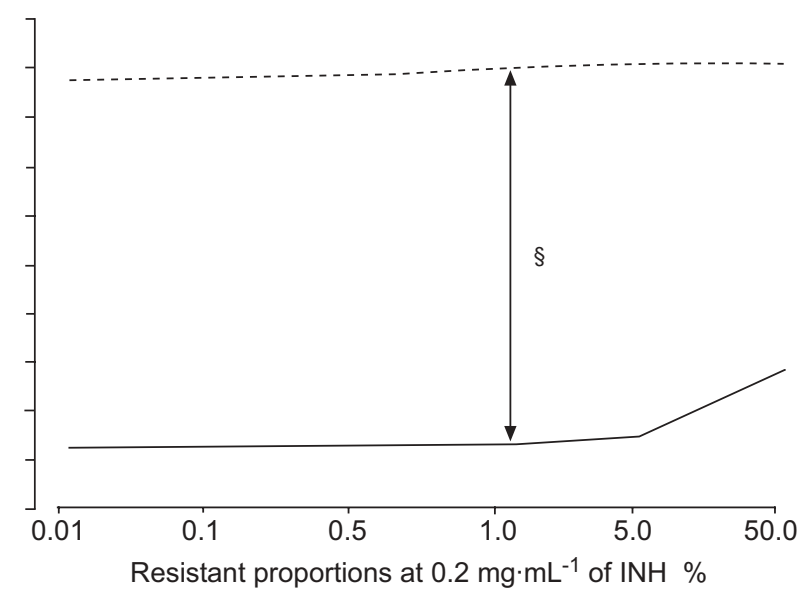

FIGURE 3. Determination of a) the critical concentration and b) the critical resistant proportion for isoniazid (INH) using probably susceptible (-----; $\mathrm{n}=99)$ and probably resistant strains (solid line; $\mathrm{n}=117$ ). MIC: minimum inhibitory concentration. ${ }^{\#:}: 13.1 \%$ as resistant; ${ }^{\circ}: 75.8 \%{ }^{+}{ }^{+}: 11.1 \%$ as susceptible; ${ }^{\text {s: }}$ 77.1\%. Data taken from [23]. 


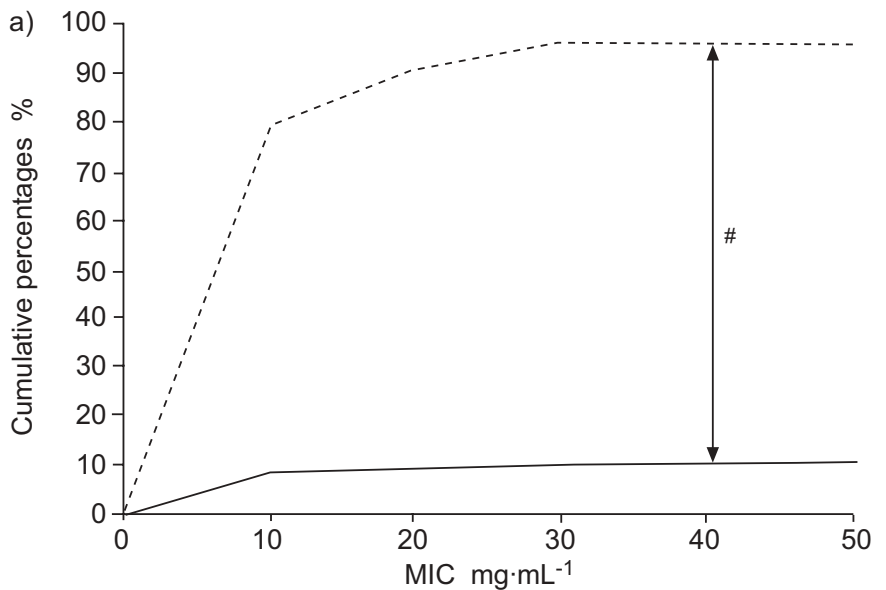

b)

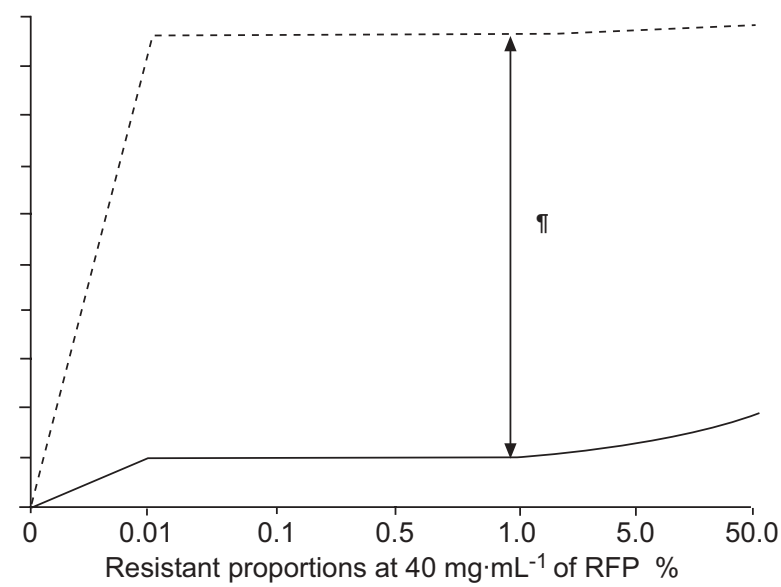

FIGURE 4. Determination of a) the critical concentration and b) the critical resistant proportion for rifampicin (RFP) using probably susceptible (-----; $\mathrm{n}=99)$ and probably resistant strains (solid line; $\mathrm{n}=108$ ). MIC: minimum inhibitory concentration. \#: 85.8\%; ": 85.6\%. Data taken from [23].
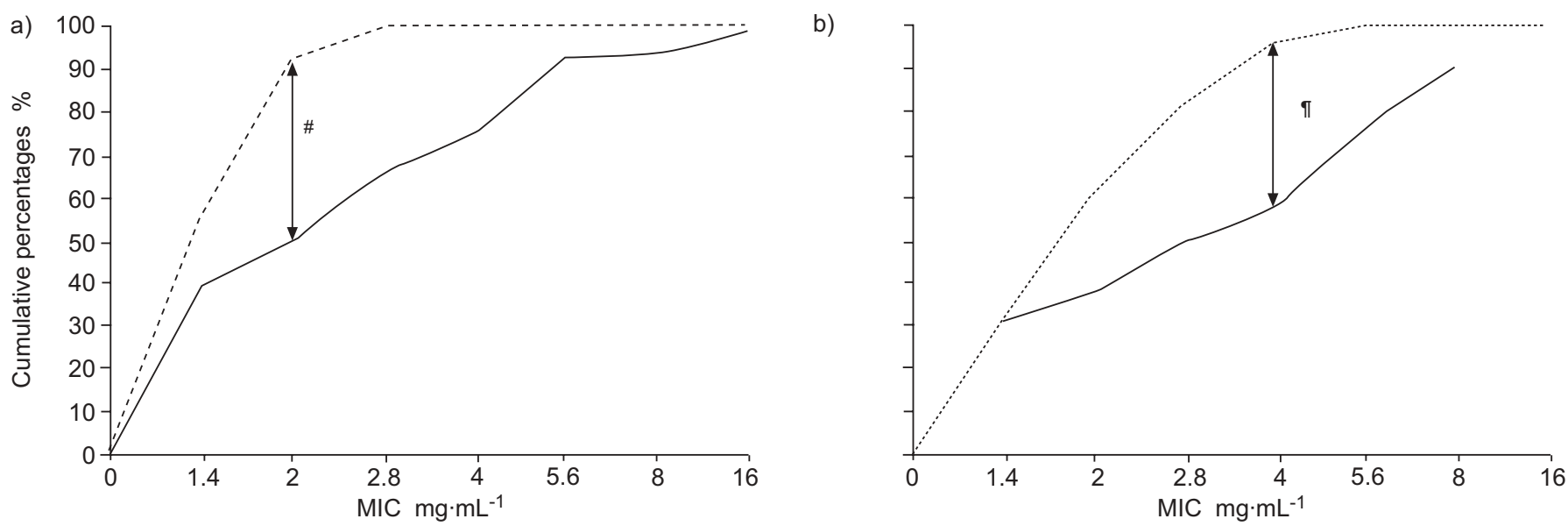

FIGURE 5. Comparison between the ethambutol critical concentrations observed at the laboratories of a) the Korean Institute of Tuberculosis, Seoul, South Korea (probably susceptible Korean strains: -----, $n=104$; probably resistant British strains (BPR): solid line, $n=124$ ), and b) the Royal Postgraduate Medical School, London, UK (probably susceptible Hong Kong strains: $\cdots \cdot . \cdot n=333$; BPR: $n=106$ ), applying the same method. MIC: minimum inhibitory concentration. \#: 41.9\%; ${ }^{*}: 38.8 \%$. Data obtained from a personal communication with D.A. Mitchison (Medical Research Council Unit for Laboratory Studies of Tuberculosis, Royal Postgraduate Medical School, Hammersmith Hospital, London, UK).

A recent survey on the current practices for DST to second-line drugs at 10 Supranational Reference Laboratories revealed important differences with regards to the critical concentrations of drugs and the critical proportions of resistance [25], underlining the need to standardise the methods and criteria with the purpose of optimising the clinical relevance of DST results.

Development of drug resistance by selective multiplication of resistant mutants existing in a wild bacillary population is determined by the initial size of actively multiplying organisms in the lesions, the drug exposure time and the drug concentration $[4,24,26,27]$. The absorption, the diffusion into the lesions and the maintenance level of a drug are all important factors for the emergence of drug resistance. INH and RFP, whose susceptibility testing results are fairly reliable, show a peak serum concentration over 100-times higher than the MIC, and it is certainly possible to maintain them at a fairly high concentration in the lesions throughout the treatment, unless the patient interrupts drug intake (fig. 6) [27, 28]. Conversely, peak serum levels of cycloserine, EMB, ciprofloxacin, ofloxacin and ETH are closer to MICs (fig. 6). As a consequence, in some patients, the period of inhibitory concentration of the drug within the lesions may be short and/or the drug level may remain subinhibitory during most of the time. In general, DST results to the latter drugs display low reliability.

Many of the newly developed DST methods have not been carefully calibrated to determine in vitro criteria of resistance with appropriate clinical samples, representative of the patient's clinical outcome under the current clinical settings; instead, these criteria have often been arbitrarily chosen using laboratory strains. Unless a careful calibration is carried out, the clinical relevance of DST results remains uncertain.

Reproducibility of in vitro DST results is influenced to a great extent by the physicochemical environment of the test $[4,28]$, which requires appropriate standardisation and control of the 


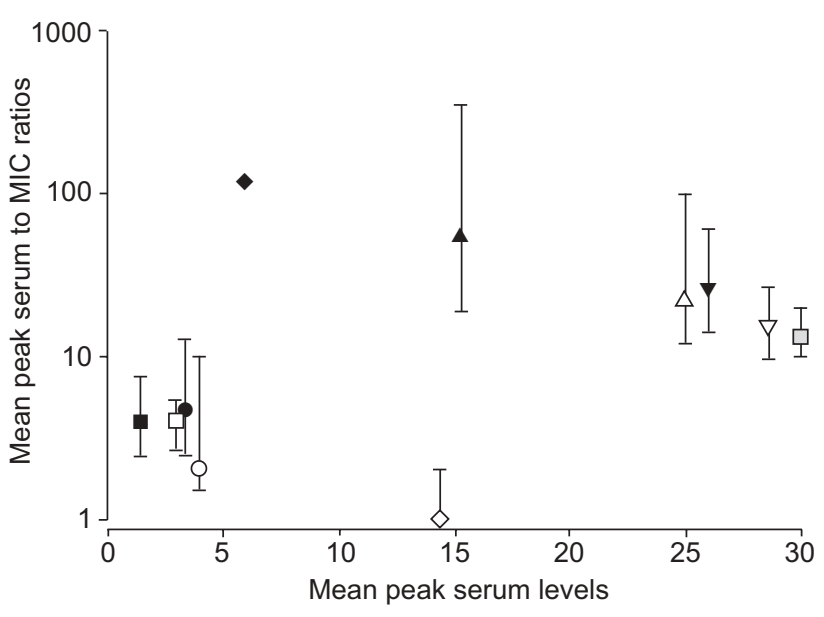

FIGURE 6. Approximated mean peak serum to minimal inhibitory concentration (MIC) ratios of antituberculosis drugs. Data are presented as mean (high/low). $\mathbf{\square}$ : ciprofloxacin; $\square$ : ofloxacin; $\bullet$ : ethionamide; $\bigcirc$ : ethambutol; $\bullet$ : isoniazid; $\diamond$ : cycloserine; $\boldsymbol{\Delta}$ : rifampicin; $\triangle$ : streptomycin; $\mathbf{\nabla}$ : para-aminosalicylic acid; $\nabla$ : capreomycin; : kanamycin. Data taken from [27, 28].

test procedure. Poor reproducibility usually stems from the procedural complexity; thus, it can be improved through procedural simplification.

Appropriate standardisation of inoculum preparation is an important prerequisite to obtain reliable DST results, since the number (size), dispersion and viability of the organisms in the inoculum have a significant effect on DST results. Frequent subcultures may distort drug-susceptibility levels and patterns of strains, when compared with those from the primary cultures. Culture media must allow an adequate growth of all the inoculated organisms; however, when they contain some antagonistic compounds or chemicals interfering with the drug action, results will be unreliable, unless these substances are removed or minimised. Variations in the concentration of the drug incorporated to the medium originate from protein binding, heat inactivation, loss of various amounts of drug during filter sterilisation, incomplete dissolution due to the use of improper solvents, inaccurate calculation of potency, and inaccurate dilution. Medium $\mathrm{pH}$, incubation temperature and incubation time are also important factors that influence DST results. A careful standardisation and the control of these testenvironment physicochemical factors are essential to achieve acceptable reproducibility of DST results.

\section{CONCLUSION}

Drug-susceptibility testing is still widely used as a tool for the selection of effective regimens to successfully treat tuberculosis patients (particularly multidrug-resistant tuberculosis), as well as for evaluation of programme efficiency and development of strategies to cope with the problem of drug-resistant tuberculosis. However, to provide reliable results, the drugsusceptibility testing method under evaluation for routine use must be carefully calibrated with representative clinical isolates of Mycobacterium tuberculosis strains, in order to determine in vitro test criteria, which could be used to predict, at best, the patient's clinical outcome. The test procedure has to then be standardised with maximal simplification to yield results with acceptable reproducibility regarding these criteria.

\section{REFERENCES}

1 World Health Organization. Anti-tuberculosis drug resistance in the world. The WHO/IUATLD Global Project on Anti-Tuberculosis Drug Resistance Surveillance (WHO/TB/97.229). Geneva, World Health Organization Document, 1997.

2 World Health Organization. Anti-tuberculosis drug resistance in the world. Report No. 2: prevalence and trends. The WHO/IUATLD Global Project on Anti-Tuberculosis Drug Resistance Surveillance (WHO/CDC/TB/2000.278). Geneva, World Health Organization document, 2000.

3 World Health Organization. Anti-tuberculosis drug resistance in the world. Third global report. The WHO/IUATLD Global Project on Anti-Tuberculosis Drug Resistance Surveillance (WHO/CDC/TB/2004). Geneva, World Health Organization document, 2004 (In press).

4 Canetti G, Fox W, Khomenko A, et al. Advances in techniques of testing mycobacterial drug sensitivity, and the use of sensitivity tests in tuberculosis control programmes. Bull World Health Organ 1969; 41: 21-43.

5 Kent TK, Kubica GP. Public health mycobacteriology. A guide for the level III laboratory. Atlanta, Center for Disease Control, 1985.

6 Hawkins JE, Wallace RJ Jr, Brown BA. Antibacterial drug susceptibility tests: mycobacteria. In: Balows A, Hausler WJ, Herrmann KL, Isenberg HD, Shadommy HJ, eds. Manual of clinical microbiology. 5th Edn. Washington DC, American Society for Microbiology, 1991; pp. 1138-1152.

7 Diaz-Infantes MS, Ruiz-Serrano MJ, Martinez-Sanchez L, et al. Evaluation of the MB/BacT mycobacterium detection system for susceptibility testing of Mycobacterium tuberculosis. J Clin Microbiol 2000; 38: 1988-1989.

8 Bemer P, Palicova FR, Rusch-Gerdes S, Drugeon HB, Pfyffer GE. Multicenter evaluation of fully automated BACTEC Mycobacteria Growth Indicator Tube 960 system for susceptibility testing of Mycobacterium tuberculosis. J Clin Microbiol 2002; 40: 150-154.

9 Palomino JC, Martin A, Camacho M, Guerra H, Swings J, Portaels F. Resazurin microtiter plate: simple and inexpensive method for detection of drug resistance in Mycobacterium tuberculosis. Antimicrob Agents Chemotherap 2002; 46: 2720-2722.

10 Abate G, Mshana RN, Miörner H. Evaluation of a colorimetric assay based on 3-(4,5-dimethyl-2-yl)-2,5diphenyl tetrazolium bromide for rapid detection of rifampicin resistance in Mycobacterium tuberculosis. Int J Tuberc Lung Dis 1998; 2: 1011-1016.

11 Riska PF, Su Y, Bardarov S, et al. Rapid film-based determination of antibiotic susceptibility of Mycobacterium tuberculosis strains by using a luciferase reporter phage and the Bronx box. J Clin Mirobiol 1999; 37: 11441149.

12 Gali N, Dominguez J, Blanco S, et al. Utility of an in-house mycobacteriophage-based assay for rapid detection of rifampicin resistance in Mycobacterium tuberculosis clinical isolates. J Clin Microbiol 2003; 41: 2647-2649.

13 Drowart A, Cambiaso CL, Huygen K, et al. Detection of rifampicin and isoniazid resistance of Mycobacterium tuberculosis strains by particle counting immunoassay. Int J Tuberc Lung Dis 1997; 1: 284-288. 
14 Lebrun L, Gönüllü N, Boutros N, et al. Use of INNO-LIPA assay for rapid identification of mycobacteria. Diagn Microbiol Infect Dis 2003; 46: 151-153.

15 El-Haji HH, Marras SAE, Tyagi S, Kramer FR, Alland D. Detection of rifampicin resistance in Mycobacterium tuberculosis in a single tube with molecular beacons. J Clin Microbiol 2001; 39: 4131-4137.

16 Kim BJ, Lee KH, Park BN, et al. Detection of rifampicinresistant Mycobacterium tuberculosis in sputa by nested PCR-linked single-strand conformation polymorphism and DNA sequencing. J Clin Microbiol 2001; 39: 2610-2617.

17 Lin YP, Behr MA, Small PM, Kurn N. Genotypic determination of Mycobacterium tuberculosis antibiotic resistance using a novel mutation detection method, the branch migration inhibition $M$. tuberculosis antibiotic resistance test. J Clin Microbiol 2000; 38: 3656-3662.

18 Mokrousov I, Otten T, Filipenko M, et al. Detection of isoniazid-resistant Mycobacterium tuberculosis strains by a multiplex allele-specific PCR assay testing katG codon 315 variation. J Clin Microbiol 2002; 40: 2509-2512.

19 Torres MJ, Criado A, Palomares JC, Aznar J. Use of realtime PCR and fluorimetry for rapid detection of rifampin and isoniazid resistance-associated mutations in Mycobacterium tuberculosis. J Clin Microbiol 2000; 38: 3194-3199.

20 Van Der Zanden AG, Te Koppele-Vize EM, Vijaya Bhanu N, Van Soolingen D, Schouls LM. Use of DNA extracts from Ziehl-Neelsen-stained slides from molecular detection of rifampin resistance and spoligotyping of
Mycobacterium tuberculosis. I Clin Microbiol 2003; 41: 1101-1108.

21 Pfyffer GE, Bonato DA, Ebrahimzadeh A, et al. Multicenter laboratory validation of susceptibility testing of Mycobacterium tuberculosis against classical second-line and newer antimicrobial drugs by using the radiometric BACTEC 460 technique and the proportion method with solid media. J Clin Microbiol 1999; 37: 3179-3186.

22 Lefford MJ, Mitchison DA. Comparison of methods for testing the sensitivity of Mycobacterium tuberculosis to ethionamide. Tubercle 1966; 47: 250-262.

23 Korean Institute of Tuberculosis, Korean National Tuberculosis Association. Drug susceptibility testing on mycobacteria by the proportion method. Research report, 1994.

24 Mitchison DA. What is drug resistance? Tubercle 1969; 50: 44-47.

25 Kim SJ, Espinal MA, Abe C, et al. Is second-line antituberculosis drug susceptibility testing reliable? Int J Tuberc Lung Dis 2004; 8: 1157-1158.

26 Canetti G. The J. Burns Amberson lecture: present aspects of bacterial resistance in tuberculosis. Am Rev Respir Dis 1966; 92: 687-703.

27 Iwainsky H. Mode of action, biotransformation and pharmacokinetics of antituberculosis drugs in animal and man. In: Bartmann K, ed. Antituberculosis drugs. New York, Springer-Verlag, 1988; pp. 399-553.

28 Heifets LB. Drug susceptibility in the chemotherapy of mycobacterial infections. Boca Raton, CRC Press, 1991. 